\title{
Konzepte der Muße
}

Unter Mitarbeit von Joachim Bauer, Günter Figal, Sarah Gouda, Sylvaine Gourdain, Thomas Jürgasch, Roman Kiefer , Andreas Kirchner, Alexander Lenger, Minh-Tam Luong, Stefan Schmidt, Michael Vollstädt

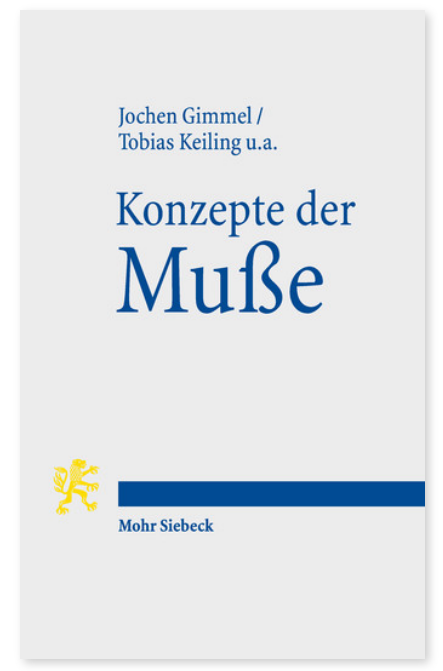

2016. VII, 104 Seiten.

ISBN 978-3-16-154987-8

DOI 10.1628/978-3-16-154987-8

eBook PDF 19,00€

ISBN 978-3-16-154648-8

fadengeheftete Broschur 19,00€
Mit einem Leben in Muße ist das Versprechen eines selbstbestimmten und erfüllten Daseins verbunden. Dabei ist keineswegs klar, was Muße ausmacht. Durch die Unterscheidung von Arbeit und Freizeit allein ist das Spezifikum der Muße nicht zu fassen. Denn Muße ist 'mehr' und anderes als Freizeit - aber was ist das für ein Mehr? Was für ein Glück verspricht die Muße? Gibt es eine Freiheit, die sich nur in Muße realisiert?

Der Band nähert sich dem Thema der Muße aus den disziplinären Perspektiven von Philosophie, Theologie, Soziologie, Psychologie und Medizin. Diese Perspektiven erschließen ein Feld von Konzepten, von denen her sich Muße verstehen lässt: Arbeit und Freizeit, Kontemplation, Gelassenheit und Achtsamkeit.

\section{Inhaltsübersicht}

\section{Einleitung}

2. Methodenprobleme

3. Konzepte der Muße

3.1. Arbeit, Freizeit und Muße

3.2. Kontemplation und Muße

3.3. Gelassenheit und Muße

3.4. Achtsamkeit und Muße

4. Problemfelder der Mußeforschung

4.1. Muße und Erkenntnis

4.2. Freiheit

4.3. Selbstbestimmung und Selbstverwirklichung

4.4. Muße und Krise

4.5. Muße als sozialstrukturelle Disposition

5. Schlussfolgerungen

Jochen Gimmel Geboren 1977; Studium der Philosophie, Soziologie und Historischen Anthropologie; 2013 Promotion; Wissenschaftlicher Mitarbeiter im Sonderforschungsbereich 1015 »Muße. Grenzen, Raumzeitlichkeit, Praktiken« (Freiburg). https://orcid.org/0000-0001-7638-8687

Tobias Keiling ist wissenschaftlicher Mitarbeiter am Institut für Philosophie der Universität Bonn; 2019/2020 Feodor LynenForschungsstipendiat der Alexander von Humboldt-Stiftung am Somerville College der Universität Oxford. https://orcid.org/0000-0001-6076-8431

Joachim Bauer Geboren 1951; Studium der Medizin in Freiburg i. Br.; Begleitend zur klinischen Ausbildung (Innere Medizin, Psychiatrie, Psychotherapie) jahrzehntelange Forschungstätigkeit, zunächst im Bereich Immunologie, dann Neurowissenschaften; längere Aufenthalte in den USA (u. a. am Mt. Sinai Medical Center in NYC); Auszeichnung mit dem Forschungspreis der Deutschen Gesellschaft für Biologische Psychiatrie; Buchautor (u. a. »Das Gedächtnis des Körpers«, »Warum ich fühle was du fühlst«, »Prinzip Menschlichkeit«, »Arbeit«, »Schmerzgrenze« und »Selbststeuerung«); seit 1992 Professor am Universitätsklinikum Freiburg; Teilprojektleiter im Sonderforschungsbereich 1015 »Muße. Konzepte, Räume, Figuren".

Günter Figal Geboren 1949; 1989-2002 Professor für Philosophie an der Universität Tübingen; 2002-17 Professor für Philosophie an der Universität Freiburg i.Br.; seit 2017 emeritiert.

Sarah Gouda Geboren 1988; Studium der Psychologie in Kairo und Freiburg; 2009 BA an der American University in Cairo; 2010 Diplom der Angewandten Psychologie an der Universität Kairo; 2012 M.Sc. an der Albert-Ludwigs-Universität Freiburg; Wissenschaftliche Mitarbeiterin im Sonderforschungsbereich 1015"Muße. Konzepte, Räume, Figuren".

Sylvaine Gourdain Geboren 1984; Studium der Germanistik und Philosophie in Frankreich und Deutschland; 2008 MA an der Université Paris-Sorbonne; 2015 Promotion zum Dr. phil. (Philosophie und Germanistik) an der Université Paris-Sorbonne und an der Albert-Ludwigs-Universität Freiburg; Forschungsstipendiatin »Move-in Louvain« am Centre Prospéro der Université Saint-Louis Bruxelles.

Thomas Jürgasch Geboren 1978; Studium der Theologie und Philosophie in Freiburg und Oxford; 2010 Promotion; seit 2020 Juniorprofessor für Alte Kirchengeschichte (Tübingen); Teilprojektleiter im Sonderforschungsbereich 1015 »Muße. Grenzen, Raumzeitlichkeit, Praktiken« (Freiburg). 
Roman Kiefer Geboren 1982; Studium der Soziologie und Neueren und Neuesten Geschichte in Freiburg, Basel und Sevilla; 2013 MA; Wissenschaftlicher Mitarbeiter im Sonderforschungsbereich 1015 »Muße. Konzepte, Räume, Figuren".

Andreas Kirchner Geboren 1983; Studium der Philosophie und Katholischen Theologie/Religionsgeschichte in Dresden und Freiburg; Doktorand im Sonderforschungsbereich 1015 Muße an der Universität Freiburg i.Br.; 2017-22 Postdoktorand an der Universität Freiburg i.Br.

https://orcid.org/0000-0002-7329-6821

Alexander Lenger ist Professor für Soziologie an der Katholischen Hochschule Freiburg.

Minh-Tam Luong Geboren 1985; Studium der Psychologie in Berlin und Toronto; 2012 Diplom an der Freien Universität Berlin; Wissenschaftliche Mitarbeiterin im Sonderforschungsbereich 1015 »Muße. Konzepte, Räume, Figuren".

Stefan Schmidt Geboren 1967; Studium der Psychologie in Konstanz und Freiburg; 2002 Promotion; 2009-11 Heymans Professor on Exceptional Experiences, University for Humanistics, Utrecht, NL; 2010-16 Juniorprofessor für Transkulturelle Gesundheitswissenschaften, Europa-Universität Viadrina, Frankfurt (Oder); seit 2006 Leiter der Sektion Komplementärmedizinische Evaluationsforschung, Klinik für Psychosomatische Medizin, Universitätsklinikum Freiburg; Teilprojektleiter im Sonderforschungsbereich 1015 »Muße. Konzepte, Räume, Figuren".

Michael Vollstädt ist Projektkoordinator der Gründungs-Akademie der Universität Freiburg.

Jetzt bestellen:

https://mohrsiebeck.com/buch/konzepte-der-musse-9783161549878?no_cache=1

order@mohrsiebeck.com

Telefon: +49 (0)7071-923-17

Telefax: +49 (0)7071-51104 